\title{
Changes in the Disease Spectrum of Hospitalized Children in a County Hospital of China
}

\author{
LIU Yong Lin, LIU Juan, SHAMSI Bilal Haider, HAO Xiao Xia
}

Department of Paediatrics, Shenmu Hospital, Shenmu County, Yulin City, Shaanxi Province, PR China.

\section{Address for correspondence:}

Bilal Haider Shamsi, LIU Yong Lin

Department of Paediatrics,

Shenmu Hospital, Shenmu County, Yulin City, Shenmu 719300, Shaanxi Province, PR China. E-mail: drhydi@gmail.com, lylsmxyy@163.com

Acknowledgements: None

Funding: Nil

Conflict of Interest: None

Permission from IRB: Yes

Ethical dilemmas faced during study: None

\section{How to cite}

LIU Yong Lin, LIU Juan, SHAMSI Bilal Haider, HAO Xiao Xia. Changes in the Disease Spectrum of Hospitalized Children in a County Hospital of China. J Nepal Paediatr Soc 2016;36(1):19-23.

doi: http://dx.doi.org/10.3126/jnps.v36i1.13632

This work is licensed under a Creative Commons Attribution 3.0 License.

\section{(c) (i)}

\begin{abstract}
Introduction: Considering the present situation of disease spectrum changes, it is very important to understand the changes of disease in primary settings, to find out the feature of disease area, to direct the decision making so as to allocate the medical resources accordingly. The objectives of this study were to understand the changing trends in disease spectrum and mortality rate of children hospitalised in the department of paediatrics in Shenmu hospital, and to provide scientific basis for the future medical care and prevention of childhood diseases. Material and Methods: A retrospective statistical analysis was done using the data regarding hospitalization of children in Shenmu County. The study lasted fifteen years, from January 1998 to December, 2012. Results: A total of 23,448 children were hospitalized below the age of 14 . Majority of the patients were younger than three years old constituting $78.8 \%$ (which included age 1 month 1 year $57.2 \%$ ). The average number of days of hospitalization was 6 8 days, with a median time of six days. The leading cause of hospitalization was respiratory disease. Other major illnesses causing hospitalization included: infectious diseases, digestive tract diseases, neonatal diseases and nervous system diseases. A total of 144 children died during this period. The top three causes of death were: asphyxia, premature birth and neonatal respiratory distress syndrome. Conclusion: The changing trends in paediatric disease hospitalisations and mortality rate over the 15 years are significant for clinicians so as to prevent and minimise such diseases by appropriate preventive and therapeutic options to contain them.
\end{abstract}

Key words: Paediatric diseases, hospitalized children, disease spectrum, childhood mortality, Chinese children.

\section{Introduction}

A ccording to literature, currently there are only a few reports about disease spectrum in paediatrics from China, except from some secondary level hospitals in county. A majority of studies about disease spectrum focuses on third class A level hospital ${ }^{1}$. It is very important to understand the changes of disease in primary setting, to 
grasp the image of disease spectrum, to find out the feature of disease area, to direct the decision making and to allocate the medical resources ${ }^{2}$.

Shenmu County in northwest Shaanxi Province is the first large coal production county in China, covers 7,635 square kilometres, has a population of 420,000 people, is the only county implementing "free medical care for all residents" from 2009 in China. In 2014, Shenmu had 353 medical and health institutions, including 21 hospitals, 27 institutes, 58 clinics, 247 village clinics. There are a total of 2,458 beds, with a 5.8 beds per one thousand people. The county has a total of 3,153 medical personnel, with a 7.5 medical personnels per one thousand people ${ }^{3}$. Shenmu Hospital is the largest and the most powerful one among other hospitals.

The aim of this study is to analyse the changes of disease spectrum of hospitalized children in our hospital from 1998 to 2012.

\section{Material and Methods}

Shenmu county hospital is the only second Class A Level Hospital of Shaanxi Province. The department of paediatrics is divided into neonatology department and general paediatrics. There are 24 beds in 1998, 36 in 2006, 54 in 2009 and 61 in 2011. Shenmu county hospital provides"free medical service to all the people, which covers more than $99 \%$ people of the county ${ }^{4,5}$.

We collected the information of hospitalized children at the age of 14 , who were admitted to the hospital from $1^{\text {st }}$ January 1998 to $31^{\text {st }}$ December 2012. All recorded data regarding: sex, age, discharge diagnosis, date and time of admission, date and time of discharge and other information were taken for this study.

If the same children came to the hospital within one year for following up, we recorded them as the same. If the child had multiple disease spectrum, then we recorded as the first diagnosis before discharge disease classification was as per the WHO guidelines ${ }^{6}$.

Statistics were analysed on the base of disease assortment and case fatality rate and changing trend of all the hospitalized children. We chose ratio or constituent ratio to indicate the enumeration data of sex, classification of diseases and death toll. We used the Pearson $\mathrm{X}^{2}$ text to compare interclass variance and also used median number, ranging (including mean and standard deviation) to show measurement data of abnormal distribution such as length of stay. Nonparametric Kruskal-Wallis rank sum test was also used. $p$-value $\leq 0.05$ indicated that the diversity had statistical significance.

\section{Results}

During the study period between1998 and 2012, we had 23,448 hospitalized children who were under 14 years old. Most were males 17,173 (73.2\%) and 6,275 (26.8\%) females. From the perspective of age, 3,009 children were under 28 days, 10,400 children were between 29 days and one year, 5,065 children were between one year and three years, 2,746 children were between three years and six years and 2,228 children were over six years age. The average length of stay in hospital was 6.8 days and intermediate was 6 days.

On divided the leaving time into three time quantum including 1998-2002, 2003-2007 and 2008-2012, we found that the portion of male children increased steadily $\left(X^{2}=25.20, p<0.01\right)$, so did the portion of young children. But at the same time, the length of staying in hospital declined steadily (Kruskal-Wallis $X^{2}=179.93, p<0.01$ ) as seen in Table 1.

The leading cause of hospitalization was respiratory system disease from beginning to end. It accounted for $49.2 \%$ in 1998-2002 and rose to $61.3 \%$ 2008-2012. Other major illnesses causing hospitalization included: infectious diseases, digestive tract diseases, neonatal diseases and nervous system diseases. The following graph shows the situation and changes of top ten diseases.

The number of death was 144 and the case fatality rate was $0.61 \%$ which was $1.04 \%(35 / 3354)$ in 1998 2002 and it decreased to $0.63 \%(50 / 7942)$ in 2003 2007. Then it further decreased to $0.49 \%(59 / 12152)$ in 2008-2012. There was a trend that the case fatality rate declined gradually $x^{2}=13.46, p<0.01$. The top three causes of death of children were asphyxia, premature birth and neonatal respiratory distress syndrome. The next two diseases were pneumonia and congenital diseases. 
Table 1: The basic information of hospitalized children in 1998-201

\begin{tabular}{lcccc}
\hline & $\mathbf{1 9 9 8 - 2 0 0 2}$ & $\mathbf{2 0 0 3 - 2 0 0 7}$ & $\mathbf{2 0 0 8 - 2 0 1 2}$ & Total \\
\hline Number of people, $\mathrm{N}$ & 3354 & 7942 & 12152 & 23448 \\
\hline Male, $\mathrm{N}(\%)$ & $2371(70.7)$ & $5743(72.3)$ & $9059(74.5)$ & $17173(73.2)$ \\
\hline Age group, $\mathbf{N}(\%)$ & & & & \\
\hline Age $\leq 28$ & $284(8.5)$ & $848(10.7)$ & $1877(15.4)$ & $3009(12.8)$ \\
\hline 29 days-1year & $1273(38.0)$ & $3855(48.5)$ & $5272(43.4)$ & $10400(44.4)$ \\
\hline 1 year-3 years & $639(19.1)$ & $1503(18.9)$ & $2923(24.1)$ & $5065(21.6)$ \\
\hline 3 years-6 years & $529(15.8)$ & $876(11.0)$ & $1341(11.0)$ & $2746(11.7)$ \\
\hline$>6$ years & $629(18.8)$ & $860(10.8)$ & $739(6.1)$ & $2228(9.5)$ \\
\hline Hospital stays & & & & $6(4-8)$ days \\
\hline Median (range interquartile) & $6(7-9)$ days & $5(7-8)$ days & $5(7-8)$ days & $6.8 \pm 3.8)$ days \\
\hline Mean value \pm standard deviation & $(7.8 \pm 4.0)$ days & $(7.2 \pm 3.5)$ days & $(2.5 \pm 3.2)$ days & $(6.8 \pm 3.5$ \\
\hline
\end{tabular}

Table 2: Showing disease spectrum of children

\begin{tabular}{lcc|lcc|lccc}
\hline & 1998-2002 & & \multicolumn{3}{c}{ 2003-2007 } & \multicolumn{3}{c}{$\mathbf{2 0 0 8 - 2 0 1 2}$} \\
\hline Diseases & $\mathrm{N}$ & $\%$ & Diseases & $\mathrm{N}$ & $\%$ & Diseases & $\mathrm{N}$ & $\%$ \\
\hline Respiratory & 1652 & 49.2 & Respiratory & 4809 & 60.6 & Respiratory & 7448 & 61.3 \\
\hline Infectious & 583 & 17.4 & GIT & 1502 & 18.9 & Neonatal & 2229 & 18.3 \\
\hline GIT & 347 & 10.3 & Neonatal & 808 & 10.2 & GIT & 1257 & 10.3 \\
\hline Neonatal & 278 & 8.3 & Infect & 262 & 3.3 & Nervous & 441 & 3.6 \\
\hline Nervous & 155 & 4.6 & Nervous & 243 & 3.1 & Nutrition & 176 & 1.4 \\
\hline Rheumatic & 81 & 2.4 & Rheumatic & 85 & 1.1 & Circulatory & 134 & 1.1 \\
\hline Urinary & 73 & 2.2 & Urinary & 48 & 0.6 & Rheumatic & 98 & 0.8 \\
\hline Nutrition & 50 & 1.5 & Poisoning & 39 & 0.5 & Infectious & 97 & 0.8 \\
\hline Blood & 45 & 1.3 & Blood & 35 & 0.4 & Blood & 57 & 0.5 \\
\hline Poisoning & 28 & 0.8 & Nutrition & 25 & 0.3 & Urinary & 46 & 0.4 \\
\hline Others & 63 & 1.9 & Others & 85 & 1.1 & Others & 46 & 0.4 \\
\hline
\end{tabular}

Table 3: Top ten causes of death in diseased children

\begin{tabular}{llllll}
\hline \multicolumn{1}{c}{ 1998-2002 } & \multicolumn{2}{c}{ 2003-2007 } & & \multicolumn{2}{c}{$\mathbf{2 0 0 8 - 2 0 1 2}$} \\
\hline Asphyxia & 7 & Premature & 9 & Premature & 13 \\
\hline Premature & 6 & Asphyxia & 8 & Neonatal respiratory & 8 \\
\hline Neonatal pneumonia & 4 & Neonatal pneumonia & 6 & Pneumonia with respiratory failure & 7 \\
\hline Bronchopneumonia & 3 & Bronchopneumonia & 3 & Asphyxia & 5 \\
\hline Acute leukemia & 3 & Intracranial haemorrhage & 3 & abbr CHD & 5 \\
\hline abbr CHD & 2 & Diarrhoea & 3 & Neonatal pneumonia & 4 \\
\hline Toxic bacillary dysentery & 1 & Neonatal septicaemia & 2 & Diarrhoea & 3 \\
\hline $\begin{array}{l}\text { Hypoxic-ischemic } \\
\text { encephalopathy }\end{array}$ & 1 & Gastrointestinal & 2 & Neonatal shock & 1 \\
\hline Neonatal septicemia & 1 & Viral encephalitis & 2 & Neonatal disseminated intravascular & 1 \\
\hline Systemic Lupus Erythematosus & 1 & Bronchitis & 1 & Neonatal septicemia & 1 \\
\hline
\end{tabular}

\section{Discussion}

There were more male patients than females amongst hospitalized children during the 15 years' span. Among them, there were 12, 173 males and 6, 275 females. Male: female ratio was 193.99:100. The statistics show that the ratio of male patients increased steadily $\left(X^{2}=25.20, p<0.01\right.$. It could have been due to the following factors: 1$)$ different gender had different tendency to get diseases and 2) China lost balance 
in s gender ratio because of birth control law. The fifth nationwide census showed that the gender ratio amongst new born was 106.8: $100^{7}$ which exceeded the normal range 104 107: $100^{8}$. It is the same with the study of Jian-nan Yang ${ }^{9}$, which indicated that the male consumed more medical resources than female.

The morbidity before the age of 3 years accounted for $74.61 \%$. This maybe related with the hypoplasia of the immune system which comes from the maternal antibody titre and decreases six months later. The physiological characters of infants are easy to be attacked by virus and bacterium causing respiratory and gastrointestinal diseases ${ }^{10}$.

The number of hospitalized children increased yearly, especially the number of neonatal prematurity and low birth weight children. The number of critically ill children also ascended. There came many changes in diseases spectrum with time, but the respiratory diseases remained the most common illnesses. The second most common were infectious diseases, digestive diseases and neonatal illnesses. The causes for high number of respiratory diseases are as follows: 1) the new born baby has a weak immune system which is easy to be attacked by respiratory diseases; and 2) the weather, meteorological factors and atmospheric conditions.

The study emphasizes to focus on the control of chlamydia pneumoniae, to produce the vaccine, and to enhance the prevention and health care to decrease the morbidity. The top 10 diseases were pneumonia, diarrhoea, upper respiratory tract infection, hyperbilirubinemia, acute tonsillitis, neonatal pneumonia, acute infectious laryngitis, preterm children, and bronchitis. There was a remarkable increase in preterm and low birth weight neonates. So the key emphasis would be to enhance the perinatal management and improvement of first aid treatment. The incidence of asthma, mycoplasma pneumonia, idiopathic thrombocytopenic purpura, and epilepsy increased gradually. Critically ill children always had severe pneumonia, infectious diseases, and congenital illnesses which remained main threats to children's health ${ }^{11,12}$.

A total of 144 children died. The total mortality rate climbed to $0.61 \%$. The mortality rate showed declining trend which was related to the overall improvement of technological skills and paediatric medical care. Neonatal diseases accounted for $14.1 \%$ of the total number of paediatric hospitalizations. But neonatal mortality was the main cause of death which accounted for $64.6 \%$ of all paediatric deaths. It coincides with the report of Xiu-hong Jia (13). In order to further reduce infant mortality, improvement in the survival rate of premature children is very important ${ }^{14,15}$. In 2014, Shenmu Hospital Paediatrics department has built isolated 48 beds neonatal ward with fully equipped NICU. The government is also supporting and focusing more to strengthen disease prevention, diagnosis and treatment of children

\section{Conclusion}

With the change of social progress and environment, the disease spectrum of children has also changed. The research of disease spectrum in Shenmu County Hospital has significant meaning for us to grasp the changes of the primary hospital paediatric diseases, to further standardize the diagnosis and treatment process, and guiding the clinical practice. It is also helpful for health administrative departments to develop health reform policy and disease research.

\section{References}

1. Li J, Chen Y, Lin Y. Investigation of disease spectrum in the PICU of Shengjing Hospital of China Medical University between 2005 and 2012. Chinese J Contemp Pediatr 2013;15(06):472-6. (In Chinese)

2. Liang $Y$, Chen Z, Huang $X$, Zeng L. Diseases Spectrum Analysis of Hospitalized Children in Guangdong Province. Chinese Med Rec 2013;18(10):51-3. (In Chinese).

3. Ren-ji Z, Ying M, Jing-ping D, Qi-ming C, Changguo $\mathrm{Y}$, Mei-juan $\mathrm{W}$, et al. Analysis on status quo of health resources in Shenmu County Shaanxi Province. Chinese J Health Policy 2010;03(8):15-8. (In Chinese)
4. Goldberg MS, Burnett RT, Stieb DM, Brophy JM, Daskalopoulou SS, Valois MF, et al. Associations between ambient air pollution and daily mortality among elderly persons in Montreal, Quebec. Sci Total Environ 2013;463-464:931-42.

5. Zhang T, Mao Y, Dang J, Chang-guo Y, Chen Q, Wang $\mathrm{M}$ et al. The background and implementation plan of "Free Health Care for all Residents" in Shenmu County Shaanxi Province. Chinese J Health Policy 2010;8:6-10. (In Chinese)

6. WHO. International Statistical Classification of Diseases and Related Health Problems 10th 
Revision. http://apps.who.int/classifications/icd10/ browse/2010/en\#/. 2015.

7. Statistics NBO. Our high sex ratio at birth. China Population Today 2005;(Z1):20. (In Chinese)

8. Schacht R, Borgerhoff Mulder M. Sex ratio effects on reproductive strategies in humans. Royal Society Open Science 2015;2(1):140402.

9. Jiannan Y, Xiaolin G. Analysis 106648 disease spectrum examples of 5-14 years old hospitalized child in Sichuan Province. Chinese $J$ Health Informatics and Management. 2011;08(6):52-5. (In Chinese).

10. Xiao-bo Z, Li-juan L, Gao-li J, Chuan-kai W, Li-bo $\mathrm{W}$, Peng $\mathrm{S}$, et al. Clinical epidemiological analysis of acute lower respiratory tract infections in 1726 hospitalized infants in single center. Chin J Evid Based Pediatr 2013;6:447-52. (In Chinese)

11. Ajayan P, Krishnamurthy S, Biswal N, Mandal J. Clinical spectrum and predictive risk factors of major infections in hospitalized children with nephrotic syndrome. Indian Pediatr 2013;50(8):779-81.

12. Patra S, Rama SU, Mahimaiha J, Subramanian AP, Shankarappa RK, Nanjappa MC. Spectrum of cyanotic congenital heart disease diagnosed by echocardiographic evaluation in patients attending paediatric cardiology clinic of a tertiary cardiac care centre. Cardiol Young 2015;25(5):861-7.

13. JIA X, YANG H, ZHU S, LI J, TANG J. Analyses on spec trum of disease and cause of death in children of inpatients ( 1997-2004). Chinese J Hosp Stats 2006;25(03):213-5. (In Chinese)

14. Kaplan M, Hammerman C. Understanding severe hyperbilirubinemia and preventing kernicterus: adjuncts in the interpretation of neonatal serum bilirubin. Clin Chim Acta 2005;356(1-2):9-21.

15. Shen N, Zhu J. Neonatal disease spectrum in hospitalized children in $\mathrm{MCH}$ in Kunming. China Maternal and Child Health 2013;02:205-9. (In Chinese) 